\title{
Ontology, Causality, and Methodology of Evolutionary Research Programs*
}

\author{
Jun Otsuka
}

June 8, 2018

\begin{abstract}
Scientific conflicts often stem from differences in the conceptual framework through which scientists view and understand their own field. In this chapter, I analyze the ontological and methodological assumptions of three traditions in evolutionary biology, namely, Ernst Mayr's population thinking, the gene-centered view of the Modern Synthesis (MS), and the Extended Evolutionary Synthesis (EES). Each of these frameworks presupposes a different account of "evolutionary causes," and this discrepancy prevents mutual understanding and objective evaluation in the recent contention surrounding the EES. From this perspective, the chapter characterizes the EES research program as an attempt to introduce causal structures beyond genes as additional units of evolution, and compares its research methodology and objectives with those of the traditional MS framework.
\end{abstract}

${ }^{*}$ To appear in: Vienna Series in Theoretical Biology: Evolutionary Causation, T. Uller and K. Laland (eds), The MIT Press. 


\section{Introduction}

Disagreements and conflicts are part and parcel of scientific practice. Mutual criticisms among opposing hypotheses, interpretations, and approaches are crucial ingredients for a healthy and productive science that prevent it from lapsing into dogmatism. Some of these problems are empirical and settled by direct observations or experiments. Others are less so and concern meta-scientific beliefs about how to interpret data or conduct experiments. The recent debate (e.g., Laland et al., 2014) between the Modern Synthesis (MS) and the so-called Extended Evolutionary Synthesis (EES), I believe, falls into the latter category. This chapter aims to substantiate this claim by focusing on the ontological and methodological frameworks of evolutionary theory. From this perspective I diagnose the current controversy as stemming from dissident views on both the ontological units of evolution and the proper methodology for studying their dynamics.

By ontology I mean a set of beliefs shared (mostly implicitly) by a group of scientists about basic entities and properties thereof that populate the domain of their investigation. Any scientific theory has its ontological assumptions as to what constitute the bricks and mortar of the world-picture according to that theory. The world of classical mechanics is filled with particles having definite location and momentum. Freud postulated an unconscious $i d$, libidos, etc., as basic psychological mechanisms. These ontological postulates are deeply linked to the notion of causality. To be is to be causally effective - no empirical theory postulates the existence of a thing that would never affect or be affected by anything else, and if it posits some- 
thing it must specify how and in what way that thing is causally related to the rest of the world. A scientific theory aims to elucidate this causal structure of the world. This implies that the ontology of a theory, along with its concept of causality, prescribes scientific methodology and practice. The goal of psychoanalysis is to bring unconscious conflicts into the conscious mind through therapeutic intervention. Dalton's atomic theory led chemists to study chemical reactions in terms of combinations, separations, and rearrangements of atoms. Conversely, methodological postulates and abilities, i.e., what we can do and observe, influence our idea of what there is in the first place ("electrons exist if we can spray them," says Hacking, 1983). Thus the ontology, the concept of causality, and the methodology of a theory together determine a conceptual framework through which we see and investigate the world.

Shared ontology and methodology facilitate communication among scientists working in the same field and enable them to evaluate each other's work. The lack thereof, in contrast, often times leads to miscommunication or fierce discussions, or what Kuhn (1962) notoriously called the incommensurability between different paradigms. Even to a lesser extent, discordance in meta-scientific assumptions prevents mutual understanding and collaborations in a scientific community. This is especially so with biology, which comprises a vast variety of disciplines with different traditions, agendas, and methodologies. The spirit of the Modern Synthesis, epitomized by Dobzhansky's "nothing in biology makes sense except in the light of evolution," was to bring a unity to the heterogeneous biological sciences under the same conceptual framework of evolutionary theory. But what is evolutionary theory? 
Since Darwin, the principle of descent with modification (due to various factors, including natural selection) has been conceptualized in different, sometimes incompatible, ways, and that has given rise to debate over what really the theory of evolution really is, even among the constructors of the Modern Synthesis (Smocovitis, 1996).

As I see it, what are often at issue in these debates are the ontology and methodology of evolutionary theory. That is, the dissonance comes from different views on what count as units and causes of evolution, and how we should study their dynamics. This chapter illustrates this by analyzing meta-scientific assumptions underlying three contemporary evolutionary thoughts: Ernst Mayr's population thinking, the gene-centered view of the Modern Synthesis, and the recently proposed Extended Evolutionary Synthesis. They view evolution differently. For Mayr, it is a change in a heterogeneous population of organisms triggered by environmental factors; for the gene-centered view, it is a shift in gene frequencies resulting from the variation in their phenotypic effects; and the EES takes the whole organism-environmental complex as both units and causes of evolutionary changes. Even in cases where they refer to the same "phenomenon," its interpretation and significance depend on the conceptual framework of each tradition. I hope clarifying these ontological and methodological assumptions in detail will help to understand the source of their disagreements and to facilitate further communication and discussion among different schools of evolutionary thought.

The chapter unfolds as follows. Section 2 begins the aforementioned meta-scientific analysis by taking up Ernst Mayr's population thinking as 
the first explicit attempt to build a unified ontology and methodology for evolutionary theory. At variance with the conventional interpretation, however, I argue that it falls short of the conceptual framework for the Modern Synthesis because of its inherent inconsistency with the Mendelian, genecentered ontology. As will be discussed in Section 3, the ontological framework of the MS was completed when genes were recognized as exclusive units underlying both organismic and evolutionary phenomena. This ontological shift came to feature population genetics as the default methodology to study evolutionary change. Section 4 then characterizes the EES as an alternative to this gene-centric picture which tries to treat whole complex causal mechanisms over genotype, phenotype, and environment as extended units of evolution. I illustrate a part of this attempt using the causal graph theory, and describe some research questions suggested from this perspective. In conclusion, Section 5 summarizes the MS and the EES frameworks as competing research programs (Lakatos, 1980), and sketches challenges and tasks for both camps with a view towards generating constructive discussion and theory development in future investigations.

\section{Population Thinking}

Great scientific works do not just reveal novel phenomena or regularities in the world but they also change how we see the world itself. That was the case for Darwin's Origin, in which he not only presented an overwhelming mass of evidence and mechanisms of evolutionary changes, but also provided a new way of looking at life. Darwin's conceptual revolution was eloquently 
epitomized by Ernst Mayr as the replacement of typological thinking by population thinking. Typological thinking, according to Mayr, has been the canonical view of living things since Aristotle, and defines an organism in terms of its essence. An essence is what specifies the nature of an individual organism as a member of a certain species. It is an unchanging standard ontologically prior to any within-specific difference or variation, which is conceived only as deviation from the essence. In contrast, population thinking puts the variation as primitive; biological populations are inherently heterogeneous and there is no fixed property shared by all members of a given species. Evolution is a necessary consequence of this variability when it is heritable and related to fitness. This made the assumption of fixed type unnecessary for, or even orthogonal to, evolutionary theory. What is crucial is rather that organisms vary from each other, and that some of their variations are heritable and have fitness effects.

Population thinking is an ontological thesis. It takes organisms as ontological primitives in the sense that they are all idiosyncratic, with any apparent commonality traditionally conceived as essence or type being merely a statistical abstraction that has no real existence.

The populationist stresses the uniqueness of everything in the organic world... All organisms and organic phenomena are composed of unique features and can be described collectively only in statistical terms... Averages are merely statistical abstractions; only the individuals of which the populations are composed have reality (Mayr, 1975, p. 28). 
For Mayr, this is the only ontological doctrine compatible with the Darwinian theory of evolution. Natural selection could not occur if populations were homogeneous, and evolution would be impossible if there were fixed types. To deny population thinking, therefore, is to deny selection and evolution.

The replacement of the typological ontology by the populationist ontology is accompanied by a conceptual shift in the notion of causality and scientific methodology. For typological thinkers, the essence of a thing is also the locus of its causal power, so that behavior, properties, and regularities exhibited by an object are determined and accounted for by its internal nature or structure. Typically, such inherent regularities are investigated in experiments that attempt to control all external variables and carefully isolate the causative agent under study so that it exhibits its "own course of nature." This, according to Mayr (1961), is the canonical concept of causation and methodology in physics, chemistry, and also what he calls "functional" biology that studies proximate causes or mechanisms of biological phenomena. Population thinking, however, implies that this understanding of causality is utterly inadequate and inapplicable for the study of evolution, simply because there is no fixed essence. Evolutionary biology thus requires a different concept of causality that does not rely on essences, and that is what Mayr calls ultimate cause. Ultimate causes are historical factors; what explains the outcome of evolution, for example, the radiation of mammals during the Paleocene and Eocene, is past environmental conditions such as the extinction of dinosaurs, which itself was triggered by an asteroid impact. Mayr (1982) claims that such causal relationships are iden- 
tified not by experiments but rather by historical narratives that connect points of past singular events into a consistent sequence of causal story.

The typological/populationist dichotomy is thus closely tied to the methodological distinction of proximate/ultimate causation. The onto-methodological dichotomy served for Mayr as a criterion to distinguish what does and does not count as an appropriate study of evolution. With this standard he condemned ahistorical approaches such as the phenetic species concept or mathematical genetics as typological (Mayr, 1982, p.41), and dismissed the accumulating knowledge in molecular, developmental, and physiological biology as irrelevant to the study of evolution because they are concerned with only proximate but not ultimate causes. In contrast, natural history, which studies natural habitats of populations and historical change thereof, is expected to play the central role in identifying ultimate causes of evolutionary trajectories. Although Mayr's appraisal here might have reflected his territorial ambition to some extent (Beatty, 1994), what should be noted is that his criticism was made on a conceptual, rather than empirical, ground. Mayr did not, for example, question theoretical results in mathematical genetics or empirical findings in molecular biology; but for him they contribute little, if anything, to our understanding of evolution, as it requires a different notion of causality and methodology.

In sum, population thinking for Mayr was not just a metaphysical corollary of Darwin's theory but rather a conceptual framework that helped him to define the very discipline of evolutionary theory both ontologically (what it is about) and methodologically (how it works). The flip side of this demarcation was the ostracism or marginalization of molecular and developmental 
studies on proximate mechanisms as they were deemed irrelevant or peripheral to the study of evolution. Another important omission is Mendelian genetics. Population thinking is just Darwinian - Mayr presents it as Darwin's exclusive legacy with no mention of Mendel. This raises serious doubts as to whether population thinking serves as an adequate ontological basis for the Modern Synthesis, which by definition was the synthesis of Darwin's theory of evolution and Mendel's theory of inheritance. ${ }^{1}$ In fact, Mendelian genetics brings an inconvenient tension into population thinking by reintroducing the notion of types (allelo- or genotypes), so that studying evolution as a change in gene frequencies requires a different ontology. This is the core claim of Dawkins' gene-centered view, to which we now turn.

\section{The Gene-Centered View}

Although variation is certainly essential for natural selection as population thinkers emphasize, it is not sufficient to produce significant adaptive change. Since most variation is small, selection must be cumulative and operate for several generations to yield any notable difference. This posed a serious problem for Darwin, who postulated a sort of blending inheritance; if parents' characters "blended" in their offspring the variation in a population would quickly fade away before selection produces any significant change, making it a negligible factor of evolution. As is well known, the problem came to be settled by the integration of Mendelian genetics into

\footnotetext{
${ }^{1}$ Witteveen $(2015,2016)$ tracks down the historical process in which Mayr has appropriated, with his own twist, the type/population distinction from its earlier articulation by Simpson and Dobzhansky.
} 
Darwin's theory (Provine, 2001), but this has also reintroduced the notion of type into evolutionary thinking by reducing organismal features into exchangeable and infusible genes. The genetic reduction was completed by Fisher's (1918) seminal work, which showed that even apparently continuous variation can be expressed as differential combinations of alleles which are themselves discrete. The Darwinian notion of a population of heterogeneous individual organisms was thus replaced by the concept of a gene pool, which consists of relatively few kinds of genotypes or allelotypes.

Through the 20th century the gene-centered view has further been buttressed by the works of T. H. Morgan, W. D. Hamilton, and G. C. Williams, to name a few, but it was Richard Dawkins who emphasized the conceptual significance of the discrete inheritance for adaptive evolution and erected it into the ontological basis of evolutionary theory. Although he does not mention it explicitly, Dawkins' target was the populationist-like ontology. Population thinking stressed the uniqueness of individual organisms as its fundamental axiom. Dawkins accepts this premise, but concludes that because of this uniqueness adaptive evolution cannot occur at the individual level. "Each individual is unique. You cannot get evolution by selecting between entities when there is only one copy of each entity!" (Dawkins, 1976, p. 34). Most selective pressure is weak, so if it is to produce a significant adaptation a population must undergo multiple rounds of selection and the result of each round must accumulate. This requires units of adaptation to be persistent and to exert a constant fitness effect across generations. Whereas individual organisms, being idiosyncratic and ephemeral, cannot do this job, genes responsible for phenotypic differences are "virtually im- 
mortal" and can serve as bearers of cumulative adaptation. Hence it is genes that evolve in the first sense, while changes in individual phenotypes are epiphenomena of the underlying genetic evolution.

Dawkins describes himself as offering "a particular way of looking at animals and plants" (Dawkins, 1982, p. 1). I would call it an ontology, for the claim really concerns what kinds of entities make up the living world. The fundamental building blocks of evolution in his view are called active germ-line replicators. They play two important roles in adaptive evolution; namely, i) to form a possibly infinite lineage of identical copies (replicas), making themselves a bearer of cumulative adaptation, and ii) to exert constant causal effects on phenotype and fitness, thereby influencing their probability of being copied. Note that these are both important characteristics of what philosophers call "type." A type is characterized by its identical nature and causal power; e.g., every instance of gold is assumed to have the same atomic structure and chemical activity, and such an in-class homogeneity is what makes gold a chemical type. Likewise, every instance of an active germ-line replicator is identical by descent, and assumedly has the same fitness effect if put in the same environmental condition. Adaptive evolution is understood as a race among such types, each of which increases or decreases its share as a consequence of its type-specific fitness effect. ${ }^{2}$

The gene-centered ontology does not leave the notion of evolutionary cause intact. The focus on the gradual and cumulative nature of adaptive

\footnotetext{
${ }^{2}$ It is no wonder, therefore, that the controversy over the gene-centered view has focused on these two alleged features of gene-as-type. The notion of identical genes must be reconciled with mutation and recombination (Dawkins, 1982, ch. 5), whereas the assumption of constant phenotypic effect has been criticized as being untenable on the face of gene-by-gene or gene-by-environment interactions (Wimsatt, 1980; Lloyd, 1988).
} 
evolution pushes historical and/or environmental conditions to the background and instead features genes as a major driving force of evolutionary changes. An active replicator "exerts phenotypic power over its world" (Dawkins, 1982, p. 91) - ADH1B allele enables rapid alcohol breakdown and the $S D$ gene has a capacity to kill flies' sperms that do not carry its copy to favor its own transmission — and by doing so they affect their frequency in future generations. The study of evolutionary dynamics, then, boils down to an analysis of the "phenotypic power" of the underlying gene. ${ }^{3}$ Each allele has its own capacity — presumably thanks to its nucleotide sequence and place within the genome - to produce a phenotype, to segregate, to mutate, to recombine, etc. Population genetics quantifies manifestations of such capacities in a given environmental condition and deduces equations that describe evolutionary dynamics under that environment. In this sense, the practice of population genetics is quite Aristotelian sensu Cartwright (1999): it studies the natural capacity of genes, and derives laws of evolution as deployments of their nature under a specified condition.

The gene-centered view thus presents quite a different ontology and methodology than those of population thinking. A biological population now consists of combinations of discrete types rather than heterogeneous individuals, and changes its composition as a function of the phenotypic power of such types. The redefinition of evolution as a change in gene frequencies features population genetics as the primary methodology to study evolutionary

\footnotetext{
${ }^{3}$ Note that the "phenotypic power" of a gene may be silent or neutral. In this sense, neutral evolution stands on the same, gene-centered ontology as neo-Darwinism. They disagree on the relative importance of various evolutionary forces (drift vs. selection), but share the same ontological framework.
} 
dynamics. In order for it to be regarded as a bona fide evolutionary process, any hypothesized evolutionary mechanism must be expressed in terms either of the phenotypic effect of genes or of environmental conditions, and its evolutionary significance must be evaluated with an appropriate populationgenetic model. That was how natural selection and drift became accepted as major forces of evolutionary changes around a century and a half-century ago, respectively, so surely shouldn't any other hypothetical mechanism go through the same procedure? The onto-methodological framework of the gene-centered view has thus come to serve as the touchstone to distinguish what is and what is not a proper study of evolution in the Modern Synthesis framework - even to the extent that "nothing in evolution makes sense except in light of population genetics" (Lynch, 2007).

\section{The Extended Evolutionary Synthesis}

The Modern Synthesis and its underlying gene-centered ontology has proven to be a very productive framework that has promoted a host of empirical findings as well as theoretical developments including, to name a few, neutral theory and inclusive fitness theory. Its hegemony, however, has recently been challenged by those who work on varieties of evolutionary mechanisms such as developmental bias, plasticity, niche construction, or epigenetic inheritance. Growing evidence from these studies suggests the mechanisms underlying evolution are too complex and diverse to be adequately captured by the MS framework - phenotypic variations are not random but canalized through developmental processes; organisms are not just passive "vehicles" 
of adaptation but actively interact with their environment to influence their evolutionary fate; and inheritance is mediated not just by genes but also by other epigenetic, environmental, and cultural means. Some believe that these findings go beyond the scope of the traditional, gene-centered approach and necessitate a conceptual update, which they call the Extended Evolutionary Synthesis or EES (Müller and Pigliucci, 2010; Laland et al., 2015).

This plea for reform is countered by those who work on the forefront of the Modern Synthesis framework (Laland et al., 2014; Welch, 2016). They doubt the alleged "novel" evolutionary mechanisms are anything more than just "add-ons" or other fancy ways genes exerts their causal power. Developmental bias, for example, is just another name for a particular way genes get expressed, plasticity an environmental-dependent expression of genotype, niche construction a kind of "extended phenotype," and epigenetic factors are merely other gene-like entities with a peculiar transmission pattern. Construed as such, these mechanisms can be well handled by the existing population-genetic models with modest adjustments and proper parameterization. Of course these modifications should affect evolutionary trajectories, but by themselves they are not essential, or at least not anything that require a significant extension or overhaul of the current evolutionary theory, or so the skeptics argue.

The contention here is not whether these phenomena exist — both camps agree that developmental bias, niche construction, etc., are real, and maybe common, phenomena - but rather it is about their significance to evolutionary theory. The populationist thinker, for example, would dismiss these 
factors as mere variations in proximate mechanisms with no direct implication for evolutionary studies of ultimate causes. From the gene-centered perspective, they are background parameters regulating genes' actions and whose evolutionary significance is evaluated only in terms of genetic changes. Proponents of the EES resist these interpretations on two fronts. First, they deny the sharp distinction between proximate/ultimate causation in favor of "reciprocal" relationships between development and evolution (Laland et al., 2011). Second, they argue for a holistic view of evolution where epigenome, developmental processes, and niche all count as their own evolutionary units that together constitute population dynamics irreducible to changes in gene frequencies (Laland et al., 2015). The EES claims that taken together these ideas suggest a new perspective or "conceptual framework" that has different points of emphasis and research questions than those of the Modern Synthesis framework.

At the same time, Laland and colleagues emphasize that the EES is not a refutation or replacement but an extension of the traditional view. This requires us to coordinate their reformist claims vis-à-vis the Modern Synthesis framework - what must be extended, and in what way? In what follows, I try to find answers to these questions in line with what has been developed so far, by characterizing the "extension" claimed by the EES as concerning the ontology and methodology of evolutionary theory.

\section{Extended ontology}

One of the central tenets of the EES is that mechanisms underlying evolution are more complex and diverse than the Modern Synthesis often pre- 
supposes, and that makes a difference in evolutionary trajectories. But how do proximate causal mechanisms influence ultimate evolutionary dynamics? Despite Mayr's strict segregation, the fact that the two sorts of causes are systematically related can be shown by the causal graph theory, which deals with formal relationships between a causal structure represented by a directed graph and a probability distribution generated from it (see box). The graphical representation has been used to illustrate causal assumptions underlying the standard quantitative genetic models (Frank, 1997; Rice, 2004; Otsuka, 2016a), but it also allows us to characterize "non-standard" evolutionary mechanisms as modifications to the standard causal model (Otsuka, 2015). In the extended causal models, epigenetic inheritance introduces an extra pathway between the parental and offspring phenotypes, while a niche is represented as a "contextual variable" constructed by individuals in a local population and inherited to the next generation (Fig. 2). Upon parameterization, these models induce probability distributions, from which evolutionary dynamics under each causal hypothesis can be calculated. The resulting equations unequivocally and quantitatively show that difference in the underlying mechanisms is reflected in the evolutionary change of the population.

Box: The causal reconstruction of evolutionary models is based on two assumptions. The first is the Price equation (Price, 1970), which gives the evolutionary change of any phenotypic mean between two genera- 
tions by

$$
\Delta \bar{Z}=\operatorname{Cov}\left(W, Z^{\prime}\right) / \bar{W}+\bar{Z}^{\prime}-\bar{Z}
$$

where $W, Z$, and $Z^{\prime}$ respectively denote fitness, phenotype, and the average offspring phenotypic value of each individual. The second is the assumption of the causal graph theory that the probability distribution of the population is generated from a causal model (Wright, 1920; Spirtes et al., 1993; Pearl, 2000). A causal model $\mathcal{M}=\langle\mathcal{G}, \mathcal{F}, P\rangle$ has three components: the causal or path graph $\mathcal{G}$ that qualitatively represents cause-effect relationships with directed edges, the set of structural equations $\mathcal{F}$ that specify the quantitative nature of each causal relationship in the graph, and the probability distribution $P$ over exogenous variables (those that have no causal input in the model). Fig. 1 is an example of a simple causal model of selection and reproduction in an asexually reproducing population. Given a genetic distribution $P(\mathbf{X})$ as an input, the model yields a joint distribution out of which the right hand side of the Price equation (1) can be calculated. If all the causal relationships are linear, the model in Fig. 1 yields the breeder's equation $\Delta \bar{Z}=S h^{2}$ (Otsuka, 2016a). By modifying the causal model (graph and functions) one may obtain evolutionary dynamics for different mechanisms. 


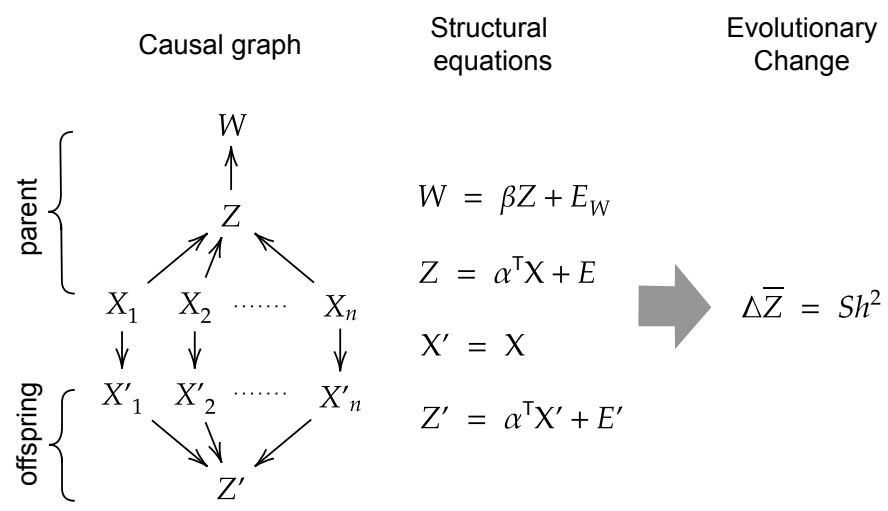

Figure 1: the causal model of the breeder's equation for an asexually reproducing population. $X \mathrm{~s}, Z$, and $W$ respectively represent allele counts, phenotype, and fitness of a parent, whereas $X^{\prime}$ s and $Z^{\prime}$ denote averages over offspring. Environmental influences $(E \mathrm{~s})$ are assumed to be independent and omitted from the graph. Parameters $\alpha$ and $\beta$ represent additive genetic effects and the selection gradient, respectively. The graph and the structural equations (in the middle) together induce a probability distribution, from which evolutionary change is calculated.

In the traditional model (Fig. 1), genes take full responsibility for both selection (as the eventual cause of fitness) and reproduction (as the sole mediator of parent-offspring resemblance). The streamlined picture justifies one to take genes as the primary causal agent of evolution, while pulling together development and other environmental factors as background conditions that regulate the genetic action (Fig. 3, left). As a natural consequence, organismic features or behaviors come to be understood as properties or capacities of a genome, e.g., niche construction as an "extended phenotype" and plas- 

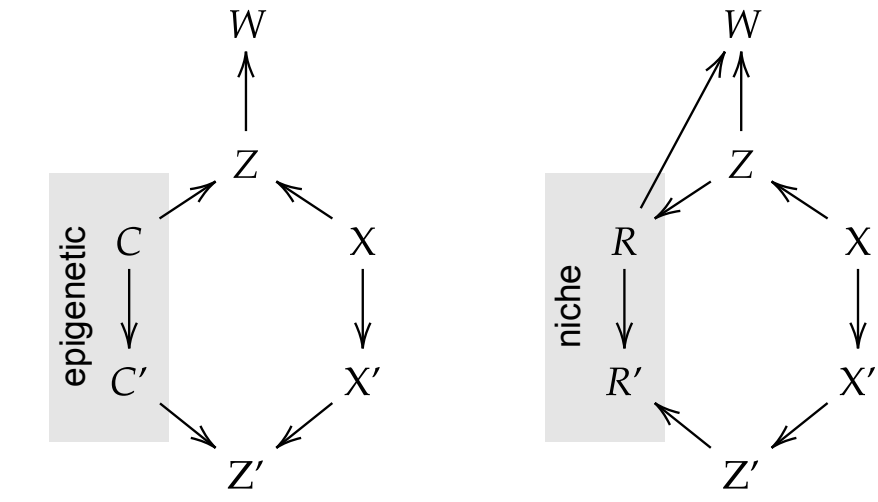

$$
\Delta \bar{Z}=\frac{1}{\bar{W}} \beta\left[\sigma_{A}^{2}+\sigma_{e p i}^{2}\right]+v\left(\bar{E}_{C}-\bar{C}\right) \quad \Delta \bar{Z}=\frac{1}{\beta \bar{Z}+R}\left(\beta+\lambda_{2}\right) \sigma_{A}^{2}
$$

Figure 2: Causal graphs and resulting evolutionary equations for epigenetic inheritance (left) and niche construction (right). The right pathway through $\mathbf{X}$ summarizes the genetic inheritance as represented in Fig. 1. The epigenetic factor $C$ and niche $R$ both create additional pathways connecting parental fitness $W$ and offspring variables $Z^{\prime}$ or $R^{\prime}$, thereby influencing adaptive dynamics. The evolutionary response under each mechanism is obtained under the assumption of linear causal relationships. See Otsuka (2015) for details.

ticity as a conditional gene expression in response to a changing environment. The gene-centric picture, however, does not fit well with the extended models where extra-genetic and environmental factors form additional causal pathways underlying selection and inheritance in parallel with the genome (Fig. 2). Here, these factors serve no longer as background parameters but rather as variables that change their distribution - that is, evolve - in response to their fitness effect. Hence, in order for a model to be dynamically sufficient and to correctly predict evolutionary change, an evolving population must be characterized not by its genetic composition alone but also 

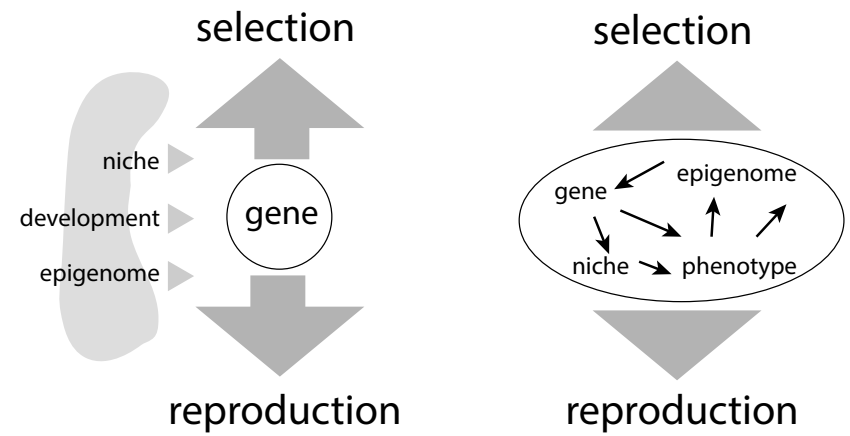

Figure 3: Schematic representations of adaptive evolution according to the MS and the EES. Left: in the gene-centered view, genes are the principal factor of both selection and reproduction, with other phenotypic and environmental factors regulating the genetic action. Right: in the extended picture, it is the whole causal structure involving genetic as well as nongenetic variables that generates evolutionary dynamics. Various developmental and environmental processes are parts or properties of this causal structure, rather than the genome alone.

by epigenetic, phenotypic, and even environmental variables related to each other via a certain causal network. This suggests an inclusive ontology that takes the entire causal structure as the generator of evolutionary dynamics and the basic unit of its analysis (Fig. 3, right). Accordingly, various evolutionary mechanisms such as niche construction or developmental plasticity are re-construed as structural properties, e.g., as a particular configuration of a causal graph or a form of structural equations.

The shift in the ontological focus from genes to structures has implications for the notion of evolutionary cause. Traditional evolutionary thinking has assumed a dualistic, figure-ground concept of causality, where the notion of causes is divided into two categories, only one of which is granted primary importance in evolutionary studies. Population thinkers set a strict 
boundary between ultimate causes of evolution and proximate mechanisms. Neo-Darwinians draw genes as the central "figure" that drives evolutionary change on the "ground" of environmental or developmental conditions. In contrast, the EES upholds an univocal and democratic view of causality, which denies the sharp distinction between proximate and ultimate causation, or between a primary causal agent and background conditions. The systematic derivation of evolutionary equations from causal models of proximate mechanisms demonstrates that proximate causes are ultimate causes of evolution (Otsuka, 2015). It also shows that evolutionary dynamics is determined not solely by a gene's capacity, but from the entire causal structure including genotype, phenotype, and environment as its parts. Of course this does not mean all these factors make an equal contribution; the genome will remain one of or perhaps the most important factor and for that reason it is privileged in studies of evolution, but its causal and evolutionary significance can be evaluated only within the network of organismal and environmental factors. Or, in other words, evolutionary change is a function not of genes alone but of the entire causal structure, so that there is no ontological justification to distinguish figure and ground in the kinds of evolutionary causes.

\section{Extended methodology}

In the Modern Synthesis framework, genes serve as the primary unit of evolutionary analysis. Models of population genetics study how evolutionary dynamics, defined in terms of genetic frequencies, arises from genes' capacities or properties under a given condition. By shifting the focus of 
analysis from genes to entire causal structures, the EES rather asks how the evolutionary dynamics of a population is generated and constrained by the underlying structure. Does this justify treating causal structures as an additional unit of evolution? The argument for genes as the primary unit derives its force from their ability to influence evolutionary processes, their stability across generations, and their changeability via mutation - these three features together qualified genes as the inductive basis of evolutionary analyses. If causal structures are to play a similar role, we must likewise ask how they affect evolution, are conserved across generations or even among species, and "mutate" and evolve through time. This prompts three research questions as described below.

Recent studies in Evo-Devo have suggested that developmental processes may bias or constrain phenotypic variability and thereby influence the rate and direction of evolutionary change (see Laland et al., 2015, and references therein). Analyzing this relationship will constitute the first line of research to evaluate the role of causal structures on evolutionary dynamics. Although short-term constraints on adaptive processes have often been studied through the (eigen) structure of the $\mathbf{G}$ matrix (e.g., Hansen, 2006; Blows, 2007), it only provides a temporary measure because genetic variation keeps changing due to selection, drift, mutation, and so on. To understand a longer-term evolvability one needs to turn to the source of these genetic variations, which is in turn determined by structural features of the underlying developmental/causal pathways. It is expected, for example, that two sets of traits will evolve independently from each other if they do not share developmental resources and form distinct modules (Wagner and Altenberg, 
1996). On the other hand, generative entrenchment contributes to the evolutionary conservatism of early developmental stages through a strong stabilizing selection on their broad downstream effect (Wimsatt and Schank, 1988). Qualitative features of developmental/causal structures thus determine both properties of the mutation matrix and selective regime, thereby influencing evolutionary dynamics. These relationships between possible phenotypic distributions and the underlying causal structure are summarized by the so-called Markov condition (Spirtes et al., 1993; Pearl, 2000, see also Fig. 4). The systematic relationship allows one to infer unknown causal structures from phenotypic records (Shipley, 2000; Valente et al., 2010; Otsuka, 2016b), or to derive qualitative predictions on long-term evolutionary trajectories from known causal structures.

The second type of research concerns extrapolations of evolutionary models from one context to another. Many models of population/quantitative genetics are local: they are built and parameterized for particular populations at a given time to track their evolutionary trajectory for a few generations. In contrast, some of our questions on evolutionary processes have more global scopes: do different populations or species of the same genus respond to selection in a similar fashion? How far can one extrapolate the result of a particular population-genetic model? Answers to these questions hinge on the stability of the underlying causal structures, with one hypothesis being that different populations (or the same population at different times) exhibit qualitatively similar evolutionary dynamics as far as they share the same causal structure. The reported similarity in the patterns of genetic and/or phenotypic correlations in related species of mammals (Young and 
(a)

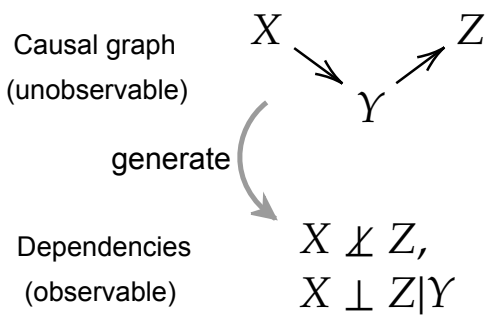

(b)

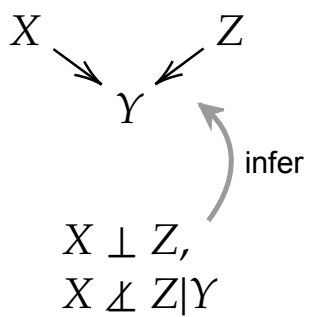

Figure 4: Although causal structures are usually unobservable, they leave some trace in the probability distribution they generate. In the above figure (a), $Z$ is expected to depend on its remote cause $X$ (i.e, $X \not \perp Z$ ) but become independent if we condition on the intermediate variable $Y(X \perp Z \mid Y)$. The Markov condition generalizes this intuition and claims that any variable in the graph becomes independent from its non-effects if conditioned on its direct causes. It turns out the same condition implies, in the case of (b), that $X$ and $Z$ are unconditionally independent but become dependent when conditioned on $Y(X \perp Z$ but $X \not \perp Z \mid Y)$. The causal search algorithms (Spirtes et al., 1993; Pearl, 2000) exploit these relationships between causality and probability to infer causal relationships among variables. 
Hallgrímsson, 2005) and of flowers (Bissell and Diggle, 2010) indicates that the conserved developmental structures generate a similar evolutionary dynamics in these populations. If this is a general phenomenon, causal models may serve as units of induction of a broader scope, unifying evolutionary dynamics of different populations.

The final, but not least important, question is how causal structures themselves evolve. Population-genetic models study changes in genetic and phenotypic frequencies under the assumption of a fixed causal structure, but from a broader perspective these causal structures are themselves products of evolution. Past studies have revealed cases of both wide-range homologies and radical changes in developmental mechanisms (e.g., True and Haag, 2001; Shubin et al., 2009). These empirical findings prompt a theoretical inquiry on laws that govern change and stasis of causal/developmental structures. Are these macro-evolutionary changes still in reach of the traditional population genetics apparatus, or do they require a new approach? In the causal modeling framework, qualitative changes in causal structure $(\mathcal{G}, \mathcal{F})$ and quantitative changes in genetic or phenotypic distribution $(P)$ are distinguished, at least notationally. Whether they represent distinct evolutionary processes, however, is a question that awaits further theoretical as well as empirical investigations. Given that developmental mechanisms are underpinned by some genes, changes in causal parameters could in principle be modeled as the epistatic evolution of regulative genes that control the strength of causal relationships (Hansen and Wagner, 2001). Although the complexity of nonlinear interactions and the sensitivity to local conditions make predictions and analytical studies along this line difficult, some general 
insights may still be obtained through simulation studies (e.g., Jones et al., 2014). Another possibility is that structural changes are caused by different kinds of genes; Carroll (2005), for example, suggests that major differences among species are attributed to variation in cis-regulatory regions, and that evolution in regulatory structure obeys different dynamics than that of the coding regions as modeled in population genetics (but see also Hoekstra and Coyne, 2007).

With these three questions the EES introduces an additional layer of research methodology, putting forward a two-story view of evolutionary theory where each level deals with different timescales, ontological units, and epistemic goals. Traditional population genetics constitutes the first layer of this extended picture which focuses on micro-scale quantitative changes in genetic and phenotypic frequencies in a fixed environment. The second layer, on the other hand, examines conditions and assumptions of these quantitative analyses by attending to the underlying causal structures and evolution thereof. It asks, for instance, how developmental homology or various types of organism-environment interactions are established and conserved, and how they affect evolutionary dynamics of local populations. The focus here is more on breadth than depth, or on qualitative characterizations of largescale evolutionary trends rather than qualitatively accurate predictions of particular populations.

The two-story methodology is a corollary of the extended ontological framework discussed above. Introducing causal/developmental structures as additional ontological units beside genes and treating developmental plasticity, inclusive inheritance, niche construction, and so on, as irreducible prop- 
erties of such units requires a matching methodology to study the causal and evolutionary implications of those units. Inversely, if these phenomena turned out to be well handled by extrapolation of the existing methodology, no addition would be needed to the ontological inventory of evolutionary theory. The extensions of the ontology and methodology of evolutionary theory are, therefore, two sides of the same coin of the proposed new "conceptual framework." From this perspective, the success of the EES depends on whether it can provide a productive and unified research program that integrates various evolutionary phenomena in a way not captured by the traditional methodology. This section has sketched a few research directions suggested from the causal modeling framework, though they are admittedly partial and possibly biased interpretations of the EES project as a whole. Unifying all these approaches under the same methodological framework is a challenging task, but will be essential for the EES to become a new synthesis of evolutionary studies.

\section{Quo Vadis?}

This chapter has compared conceptual frameworks of evolutionary theory in terms of their ontological and methodological assumptions, with a special focus on the Modern Synthesis and the Extended Evolutionary Synthesis. From this perspective, the debate between the MS and the EES can be seen as a reductionist vs. anti-reductionist tug-of-war in evolutionary biology. The MS is characterized by its reductionist tendency, trying to represent and study organismal features and evolutionary phenomena in terms of un- 
derlying genes. Genes (and other gene-like entities) are the exclusive building blocks of evolution, so that all complex evolutionary mechanisms and macro-evolution can and must be captured through elaborations or extrapolations of the current population-genetic models. Proponents of the EES contest this view by pointing out that the phenomena they call constructive development cannot be reduced to just properties of the genome. Rather, they are properties of causal structures which, as higher-level units of evolution, prompt new lines of research questions that demand an alternative approach.

Where does the debate go from here? I for one doubt there is a single or a handful of empirical litmus tests that would settle the issue once and for all. When the contention concerns different conceptual frameworks, such a Popperian falsification is the last thing we could hope for. But that does not mean we should be Kuhnian fatalists and accept that debates between two different paradigms do not come to an end until one of them dies out. A more constructive picture would be to view the MS and the EES as two competing research programs in the sense of Lakatos (1980). A research program, according to Lakatos, consists of its core tenets or "hard core" and a "protective belt" that relates the core claims to empirical data or tests. When results from the tests do not turn out as expected, or unpredicted phenomena are observed, researchers rarely give up the hard core of their theory but try to accommodate anomalies by modifying the protective belt. In this way the same research program can change and "evolve" in the face of apparent difficulties while keeping its central claim intact.

The gene-centric ontology and methodology constitute the hard core of 
the Modern Synthesis. Historically this program faced several challenges, including neutral evolution, the problem of altruism, and punctuated equilibrium, to name a few, but each time it survived to evolve by modifying its protective belt (e.g., drift as an important evolutionary process, the concept of inclusive fitness, etc.). In this sense it was a progressive program, and this history of past successes underlies the expectation of many that the current challenge from the EES can also be handled by a similar maneuver - as new "add-ons" to the same hard core. In contrast, proponents of the EES are skeptical and complain that the handling of the MS program of complex developmental and environmental interactions has begun to show a sign of degeneration, issuing ad-hoc patches just to save its core doctrines but without leading to novel research questions or predictions. What we need is not superficial treatment but major surgery of the hard-core ontology and methodology, and only within a renewed research program can we appreciate the full evolutionary implications of these mechanisms, or so they argue.

The question we should ask, therefore, is not which research program is correct, but rather which is more progressive and offers novel predictions and insights on evolution. From this perspective, the challenge for the MS is not just to show the consistency of phenomena like developmental plasticity, inclusive inheritance, etc., with its core framework, but to devise a suite of auxiliary theories that provide novel insight into these phenomena from the gene-centered perspective, just as it did in the cases of neutral theory and inclusive fitness theory. In contrast, what is needed for the EES camp is to provide effective means to bring its core tenets to actual scientific 
investigations along the direction it envisages. Provided that constructive development and reciprocal causation are ubiquitous and important, how can we forge these concepts into an empirical hypothesis that makes testable predictions? In the dawn of the Modern Synthesis, population genetics has served as a formal platform both to quantitatively verify Darwin and Wallace's idea of natural selection (which was at its time quite dubitable), and to stimulate subsequent investigations, say, on the amount and the source of genetic variation in natural populations. Can the EES provide a similar, unifying theoretical framework? Recent works on Evo-Devo, epigenetic inheritance, and complex organism-environment interactions have laid seeds of hitherto neglected or marginalized research directions; whether these seeds grow into a new progressive research program or get incorporated as an auxiliary "protective belt" of the existing one hinges on future conceptual, theoretical, and empirical investigations.

\section{Acknowledgement}

I thank Tobias Uller, Kevin Laland, an anonymous referee, and the participants of the KLI Workshop on Cause and Process in Evolution for stimulating discussions and insightful comments on the draft of this chapter. This work is supported by JSPS grant-in-aid 16K16335.

\section{References}

Beatty, J. (1994). The proximate/ultimate distinction in the multiple careers of Ernst Mayr. Biology \& Philosophy, 9(3):333-356. 
Bissell, E. K. and Diggle, P. K. (2010). Modular genetic architecture of floral morphology in Nicotiana: quantitative genetic and comparative phenotypic approaches to floral integration. Journal of Evolutionary Biology, 23(8):1744-1758.

Blows, M. W. (2007). A tale of two matrices: multivariate approaches in evolutionary biology. Journal of Evolutionary Biology, 20(1):1-8.

Carroll, S. B. (2005). Evolution at Two Levels: On Genes and Form. PLoS Biology, 3(7):e245.

Cartwright, N. (1999). The Dappled World. A Study of the Boundaries of Science. Cambridge University Press, Cambridge, UK.

Dawkins, R. (1976). The selfish gene. Oxford University Press, Oxford, UK.

Dawkins, R. (1982). The Extended Phenotype. The Long Reach of the Gene. Oxford University Press, Oxford, UK.

Fisher, R. A. (1918). The correlation between relatives on the supposition of mendelian inheritance. Transactions of the Royal Society of Edinburgh, $51: 399-433$.

Frank, S. A. (1997). The Price equation, Fisher's fundamental theorem, kin selection, and causal analysis. Evolution, 51(6):1712-1729.

Hacking, I. (1983). Representing and Intervening. Introductory Topics in the Philosophy of Natural Science. Cambridge University Press, Cambridge, UK. 
Hansen, T. F. (2006). The Evolution of Genetic Architecture. Annual Review of Ecology, Evolution, and Systematics, 37(1):123-157.

Hansen, T. F. and Wagner, G. P. (2001). Modeling genetic architecture: a multilinear theory of gene interaction. Theoretical Population Biology, $59(1): 61-86$.

Hoekstra, H. E. and Coyne, J. A. (2007). The locus of evolution: evo devo and the genetics of adaptation. Evolution, 61(5):995-1016.

Jones, A. G., Bürger, R., and Arnold, S. J. (2014). Epistasis and natural selection shape the mutational architecture of complex traits. Nature Communications, 5:3709.

Kuhn, T. S. (1962). The Structure of Scientific Revolutions. University of Chicago Press, Chicago, IL.

Lakatos, I. (1980). The methodology of scientific research programmes. Philosophical papers volume 1. Cambridge University Press, Cambridge, UK.

Laland, K. N., Sterelny, K., Odling-Smee, J., Hoppitt, W., and Uller, T. (2011). Cause and effect in biology revisited: is Mayr's proximate-ultimate dichotomy still useful? Science, 334:1512-1516.

Laland, K. N., Uller, T., Feldman, M. W., Sterelny, K., Müller, G. B., Moczek, A., Jablonka, E., and Odling-Smee, J. (2015). The extended evolutionary synthesis: its structure, assumptions and predictions. Proceedings of the Royal Society B: Biological Sciences, 282(1813):20151019. 
Laland, K. N., Uller, T., Feldman, M. W., Sterelny, K., Müller, G. B., Moczek, A., Jablonka, E., Odling-Smee, J., Wray, G. A., Hoekstra, H. E., Futuyma, D. J., Lenski, R. E., Mackay, T. F. C., Schluter, D., and Strassmann, J. E. (2014). Does evolutionary theory need a rethink? Nature, 514(7521):161-164.

Lloyd, E. A. (1988). The Structure and Confirmation of Evolutionary Theory. Princeton University Press, Princeton, NJ.

Lynch, M. (2007). The frailty of adaptive hypotheses for the origins of organismal complexity. PNAS, 104(Suppl 1):8597-8604.

Mayr, E. (1961). Cause and effect in biology. Science, 134:1501-1506.

Mayr, E. (1975). Evolution and the Diversity of Life: Selected Essays. Harvard University Press.

Mayr, E. (1982). The Growth of Biological Thought. Diversity, Evolution, and Inheritance. The Belknap Press of Harvard University Press, Cambridge, MA.

Müller, G. B. and Pigliucci, M. (2010). Evolution: the extended synthesis. The MIT Press, Cambridge, MA.

Otsuka, J. (2015). Using causal models to integrate proximate and ultimate causation. Biology \& Philosophy, 30(1):19-37.

Otsuka, J. (2016a). Causal Foundations of Evolutionary Genetics. The British Journal for the Philosophy of Science, 67:247-269. 
Otsuka, J. (2016b). Discovering phenotypic causal structure from nonexperimental data. Journal of Evolutionary Biology, 29(6):1268-1277.

Pearl, J. (2000). Causality: Models, Reasoning, and Inference. Cambridge University Press, New York, NY.

Price, G. R. (1970). Selection and covariance. Nature, 227:520-521.

Provine, W. B. (2001). The Origins of Theoretical Population Genetics. With a New Afterword. University of Chicago Press, Chicago, IL.

Rice, S. H. (2004). Evolutionary Theory. Mathematical and Conceptual Foundations. Sinauer Associates, Sunderland, MA.

Shipley, B. (2000). Cause and Correlation in Biology: A User's Guide to Path Analysis, Structural Equations and Causal Inference. Cambridge University Press, Cambridge, UK.

Shubin, N., Tabin, C., and Carroll, S. (2009). Deep homology and the origins of evolutionary novelty. Nature, 457(7231):818-823.

Smocovitis, V. B. (1996). Unifying Biology. The Evolutionary Synthesis and Evolutionary Biology. Princeton University Press, Princeton, NJ.

Spirtes, P., Glymour, C., and Scheines, R. (1993). Causation, Prediction, and Search. The MIT Press, Cambridge, MA.

True, J. R. and Haag, E. S. (2001). Developmental system drift and flexibility in evolutionary trajectories. Evolution \& Development, 3(2):109-119. 
Valente, B. D., Rosa, G. J. M., De los Campos, G., Gianola, D., and Silva, M. A. (2010). Searching for recursive causal structures in multivariate quantitative genetics mixed models. Genetics, 185(2):633-644.

Wagner, G. P. and Altenberg, L. (1996). Complex Adaptations and the Evolution of Evolvability. Evolution, 50(3):967-976.

Welch, J. J. (2016). What's wrong with evolutionary biology? Biology $\&$ Philosophy, 32(2):263-279.

Wimsatt, W. (1980). Reductionistic research strategies and their biases in the units of selection controversy. In Nickles, T., editor, Scientific Discovery: Historical and Scientific Case Studies, pages 213-259. Reidel, Dordrecht.

Wimsatt, W. and Schank, J. C. (1988). Two constraints on the evolution of complex adaptations and the means for their avoidance. In Nitecki, M., editor, Evolutionary progress, pages 231-273. University of Chicago Press, Chicago, IL.

Witteveen, J. (2015). "A temporary oversimplification": Mayr, Simpson, Dobzhansky, and the origins of the typology/population dichotomy (part 1 of 2). Studies in history and philosophy of biological and biomedical sciences, 54(C):20-33.

Witteveen, J. (2016). "A temporary oversimplification": Mayr, Simpson, Dobzhansky, and the origins of the typology/population dichotomy (part 2 of 2). Studies in history and philosophy of biological and biomedical sciences, 57(C):96-105. 
Wright, S. (1920). The relative importance of heredity and environment in determining the piebald pattern of guinea-pigs. PNAS, 6(6):320-332.

Young, N. M. and Hallgrímsson, B. (2005). Serial Homology and the Evolution of Mammalian Limb Covariation Structure. Evolution, 59(12):26912704 . 